\title{
Clinical pathology and retinal vascular structure in the Bardet-Biedl syndrome
}

\author{
Toke Bek, Thomas Rosenberg
}

\begin{abstract}
A comparative study of clinical pathology and retinal vascular structure is described as studied by vascular casting in an eye of a patient with the Bardet-Biedl syndrome. At the time of examination the eye had been almost blind for at least 4 years. The histopathological examination showed a largely uniform loss of the outer retinal layers. The gross pathological examination of the cast ocular fundus showed three distinct zones, an inner zone inside the temporal vascular arcades where retinal vessels had been cast, a mid peripheral zone with bone spicules, and a peripheral zone with neither cast vessels nor bone spicules. The findings are discussed in relation to possible pathophysiological mechanisms involved in the development of retinal dystrophy in the Bardet-Biedl syndrome.

(Br F Ophthalmol 1995; 79: 76-80)
\end{abstract}

The Bardet-Biedl syndrome (BBS) is a hereditary autosomal recessive disease characterised by retinal dystrophy, mental retardation, polydactyly, obesity, and hypogenitalism. Internal organs may also be affected, and early death may result from renal failure. One or more of the clinical features characterising the syndrome may be absent, but retinal dystrophy is a consistent finding. ${ }^{12}$ Retinal dystrophy becomes clinically manifest in early childhood, with progressive loss of visual function, leading to severe visual disability in early adolescence. $^{2-5}$ Morphologically, the retinal dystrophy is characterised by a bull's eye appearance in the macula, and in the late stages morphological signs of retinitis pigmentosa develop, with bony spicules in the mid peripheral retina.

The pathogenesis of retinal dystrophy in BBS is unknown. Histopathological studies of eyes at different stages of the disease point to a series of events with primary degeneration of photoreceptors and secondary involvement of the other retinal layers including the retinal pigment epithelium..$^{6-9}$ Clinically, the progressive loss of retinal tissue is accompanied by ophthalmoscopically visible attenuation of the retinal vessels. However, more detailed evidence about the distribution and structure of retinal vessels in BBS is yet unreported.

This paper reports a comparative study of clinical pathology and retinal vascular structure as studied by vascular casting in a case of BBS. The findings are discussed in relation to adaptive properties of retinal vascular structure and in relation to possible pathophysiological mechanisms involved in the development of retinal dystrophy in BBS.

\section{Case report}

The patient was a female, first examined by an ophthalmologist at the age of 11 years and 9 months. At that time she reportedly had been nightblind for several years, and visual acuity had gradually decreased during the 6 months preceding the examination. The ophthalmic examination showed visual acuity of $6 / 36$ in both eyes. Corneal sensibility, ocular motility, and pupil reaction were normal. Ophthalmoscopy showed optic nerve atrophy and thinned retinal arterioles.

At the age of 13 years and 6 months the patient was seen at the Danish National Eye Clinic for the Visually Impaired where the diagnosis of retinitis pigmentosa secondary to the Bardet-Biedl syndrome was established. Parental consanguinity was denied and nor were any relatives known to have had the disease. The birth of the patient had been normal. She had attended a class for retarded children and had done reasonably well in spite of the fact that reading was only possible in very bright light. Physical examination showed: height $144 \mathrm{~cm}$, weight $71.6 \mathrm{~kg}$, and severe obesity of head, trunk, and limbs. Physical development lagged behind chronological age, but mental development was within the normal range. $x$ Ray examination showed normal sella turcica and brachydactyly of hands and feet, but no supernumerous fingers or toes. Ophthalmic examination showed VA right eye $6 / 24+1 \cdot 0 \mathrm{D}$ cyl ax $90^{\circ}$, VA left eye $6 / 24+2 \cdot 0 \mathrm{D}$ cyl ax $90^{\circ}$. There was slight nystagmus. Visual field examination on an arc perimeter with white targets $10 / 300$ and $3 / 300$ was normal in both eyes. Ophthalmoscopy showed pale optic nerve heads, thin retinal vessels, bone spicules in the retinal mid periphery, and clearly visible choroidal vessels in the retinal periphery.

The patient was examined regularly over the next 6 years, during which time the visual acuity gradually decreased, the visual field narrowed, and the mid peripheral bone spicules increased. At the age of 14 years and 6 months reading capability had been almost lost, even in very bright light. At the age of 17 years visual acuity was VA right eye $<6 / 36$ $+1.0 \mathrm{D} \mathrm{sph}+1.0 \mathrm{D}$ cyl ax $90^{\circ}$, and VA left eye $<6 / 36+1.0 \mathrm{D}$ sph, $+1.0 \mathrm{D}$ cyl ax $90^{\circ}$. At the age of 21 years visual acuity had decreased to VA right eye $6 / 60$, VA left eye $1 / 60$. 


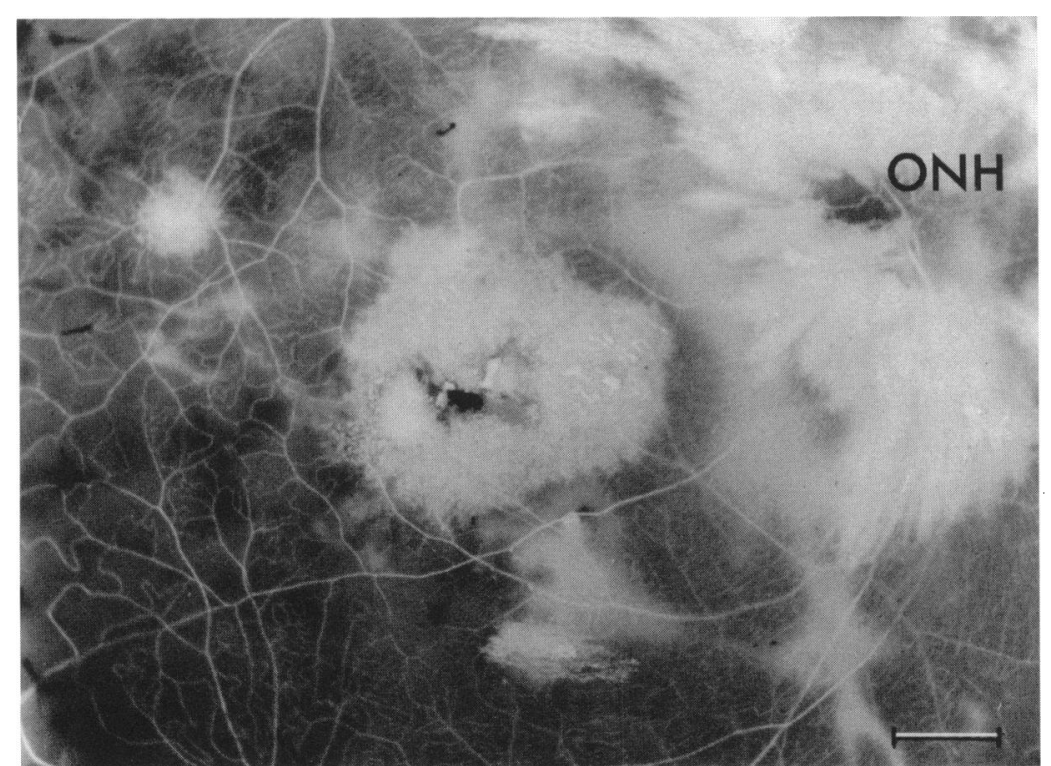

Figure 1 The central retinal zone with cast vessels. The optic nerve head (ONH) is seen to the upper right, surrounded by extensive artefactual extravasation of casting material. Similar extravasation of casting material is seen around the fovea in the centre of the figure. Vascular anastomoses between the upper and the lower temporal arcade are seen temporal from the foveal area. Bar $=1 \mathrm{~mm}$. preparation procedures, the retina was firmly adherent to the underlying choroid and displayed no tendency to detach.

\section{CASTING PROCEDURE}

The casting procedure was a slight modification of the procedure described in Bek and Jensen. ${ }^{10}$ Casting was technically successful, but increased resistance was met at the injection of casting material into the retinal vessels. After the casting procedure, the eye was immersed in a solution of equal parts $25 \%$ glutaraldehyde and $4 \%$ formalin for 20 hours. The iris and the lens were subsequently removed, and a liquified vitreous decanted. The hollow eyeball was dehydrated in ethanol, and the remaining denatured vitreous and the posterior hyaloid membrane were removed with a swab. The inner aspect of the eyeball was documented by photography on TMY $400 \mathrm{black} /$ white film (Kodak) through one of the oculars of a Zeiss SV 11 stereo microscope.

\section{HISTOPATHOLOGY}

Two horizontal slices of the eyeball were excised for histological examination, located at the level around the upper temporal vascular arcade and around the fovea, respectively. Histological sections were made parallel with the cut surface of these slices, and thus were continuous from the nasal periphery, through the macula to the temporal periphery. Successive sections were stained with haematoxylin and eosin, periodic acid Schiff with haematoxylin and toluidine blue. showed pronounced synchysis scintillan the right eye. Both eyes had maximally pale optic nerve heads, thinned retinal vessels, and peripheral hyperpigmentation indicative of retinitis pigmentosa.

During the following years, progressive renal failure with rising serum creatinine and serum carbamide developed, and the patient eventually died from uraemia at the age of 60 years 6 months. On regular admissions to hospital during the progression of renal failure the blood pressure never exceeded $135 / 80 \mathrm{~mm} \mathrm{Hg}$.

\section{Materials and methods}

TISSUE MATERIAI

The left eye of the patient was donated to the cornea bank at the Department of Ophthalmology, Århus University Hospital. The eye was small, but otherwise it appeared macroscopically normal. The cornea was clear, had normal endothelial density, and displayed no pathology that disqualified it from being used as a donor graft. The pupil was round, the iris appeared normal, and the lens was cataractous. The axial distance from the ora serrata to the macular retina could be measured with a slide gauge after the cornea, including a circular scleral rim, had been removed by trepanation. This estimate of the axial length of the eye was found to be $13 \mathrm{~mm}$. Postmortem time at fixation was 45 hours. During all

\section{Results}

\section{GROSS PATHOLOGY OF THE OCULAR}

\section{BACKGROUND}

On the basis of the morphological appearance, the ocular fundus could be divided into three concentrical zones that differed markedly from normal eyes of similar age. ${ }^{10}$

The central zone consisted of the area where retinal vessels had been cast to appear white (Fig 1). This zone comprised the macular area including the temporal arcades, extending nasally one disc diameter from the optic nerve head. The cast vessels appeared thin and displayed markedly decreased density. The normal three layered arrangement of capillaries in the macular area was absent, and only occasionally did the capillaries form two layers, mainly close to the optic nerve head where the most proximal part of the radial peripapillary capillary network was preserved. Along the temporal raphe, extensive communications were seen between the vessels originating from the upper and the lower temporal arcade. In the periphery of the zone, the cast vessels ended in terminal single layered arcades (Figs 1, 2). These arcades and other border vessels were continuous, with no abrupt closures towards the retinal mid periphery. However, in a transition zone, the cast vessels 


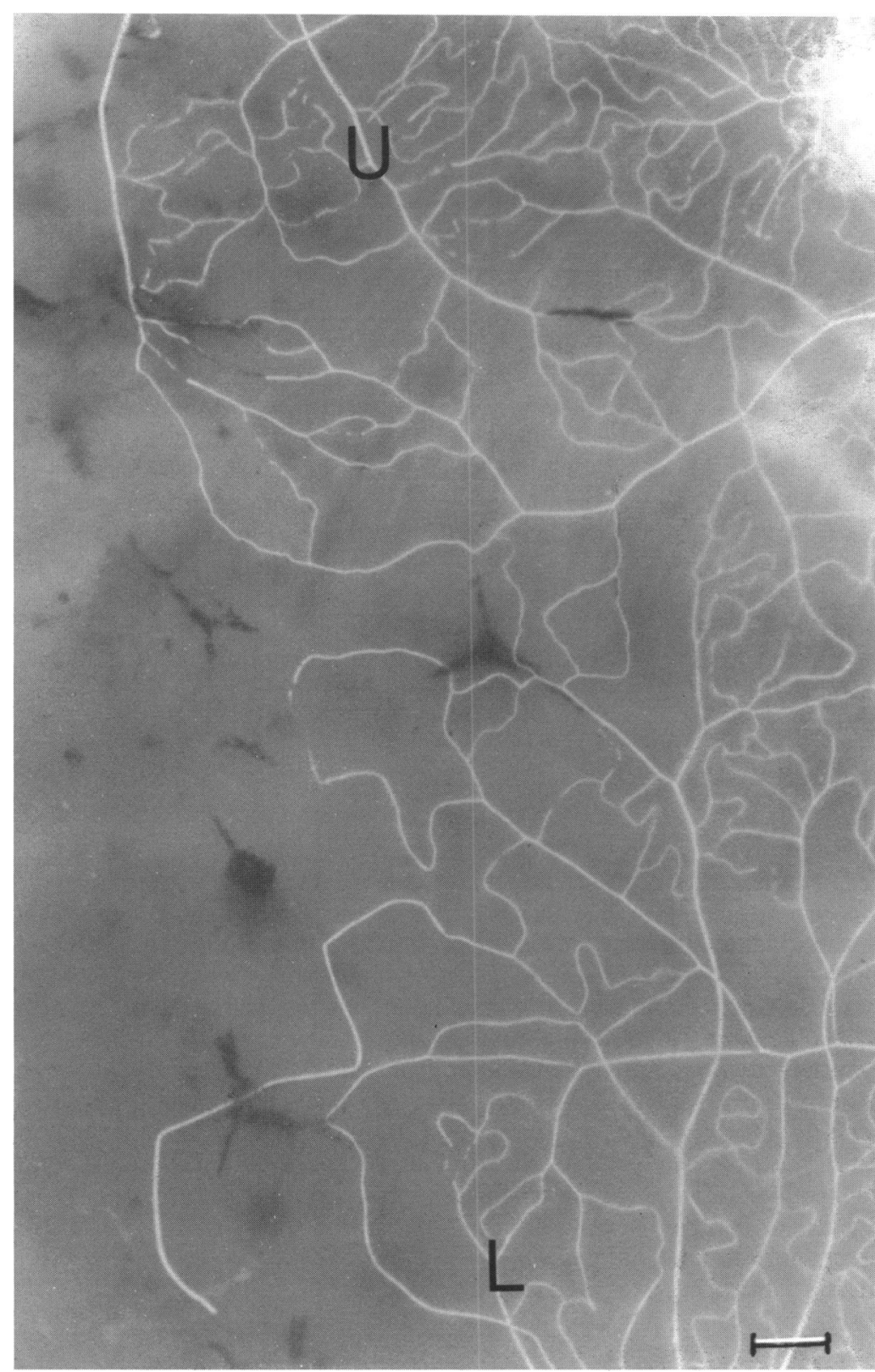

Figure 2 The temporal border of the central cast vessel area, with anastomosing terminal arcades from the upper (U) and the lower (L) temporal arcade. A few retinal vessels are seen to be ensheathed by hyperpigmented material. Bar $=250 \mu \mathrm{m}$.

were discontinuously ensheathed with hyperpigmented spicules that branched off to continue into the retinal mid periphery in radiating lines, as if representing sites of former vessels (Fig 3). A notable artefact was diffuse intraretinal extravasation of casting material at the optic disc so that its margins had become totally obscured (Fig 1). A similar pattern of diffuse extravasation of casting material was present in an area around the foveal avascular zone, thus obscuring the vessels in this area, including possible gross pathological signs of macular degeneration.

The mid peripheral zone was characterised by an abnormal accumulation of hyperpigmented bone spicules. This zone was located immediately peripheral from the central zone, as a concentric ring area with a constant width of approximately $6 \mathrm{~mm}$. Nasally, this zone almost reached the ora serrata, whereas a broader zone separated it from this margin on the temporal side. The bone spicules could be localised in two distinct layers. The spicules of the inner layers were generally long and thin, and often constituted extensions of cast vessels from the central retinal zone (Fig 3). The deeper lying spicules generally were broader and coarser, and showed no visible relation to other anatomical structures.

The peripheral zone was greyish looking with sparse bone spicules. The width of this zone varied from approximately $2 \mathrm{~mm}$ nasally to $7 \mathrm{~mm}$ temporally. The few bone spicules here were predominantly located in deeper layers, often bounding rounded stamped out lesions varying in size from 300 to $800 \mu \mathrm{m}$ (Fig 4).

Collateral filling from the injection catheter in the central retinal artery into choroidal vessels was seen close to the optic disc, and in a few vessels that radiated more distantly towards the retinal periphery.

\section{HISTOPATHOLOGICAL FINDINGS}

The histological sections showed a total lack of photoreceptors in all retinal areas. In the central and the mid peripheral retinal area the pigment epithelium was discontinuous with the cells arranged in separated islands, whereas this cell layer was continuous in most of the periphery. At places where pigment epithelial cells were present, these appeared atrophic and the outer retina had been detached from them, whereas the outer retina adhered to the underlying choroid where the pigment epithelial cells were absent (Fig 5). Bruch's membrane was uniformly thickened at all eccentricities, and no sign of the choriocapillaris could be recognised. The choroid appeared fibrotic, but larger choroidal vessels were present. In the inner retina, the inner nuclear layer and few ganglion cell bodies could be distinguished. The cell density of these layers was considerably decreased, and gliosis was prominent. Müller cell processes traversing the inner retina were clearly seen in most of the sections. Larger retinal and choroidal vessels displayed thickening of the walls but no specific hypertensive changes could be recognised.

Focal black areas appeared in two forms in the inner retina. One form consisted of casting material, either as well delimited round areas of black material representing shrunken casting material inside retinal vessels (Fig 5) or as irregular extravasated lumps. Cast vessels were almost exclusively seen around the inner plexiform layer and the ganglion cell layer, whereas a very few vascular loops were seen to take a course down towards the outer plexiform layer. In the macular area extravasated casting material was seen inside cystoid spaces in the inner retina. The other form of black material appeared as grainy aggregates of pigment (Fig 6), predominantly associated with retinal vessels, but also occurring in irregular lumps elsewhere in the retina (Fig 5). The black grains seen at the peripheral aspect of the walls of larger retinal vessels occupied the whole circumference or parts of it (Fig 6). In some instances, thickened circumferential hyperpigmentation protruded to narrow the vascular lumen appreciably. Occasionally, the vascular lumen was totally obliterated by the 


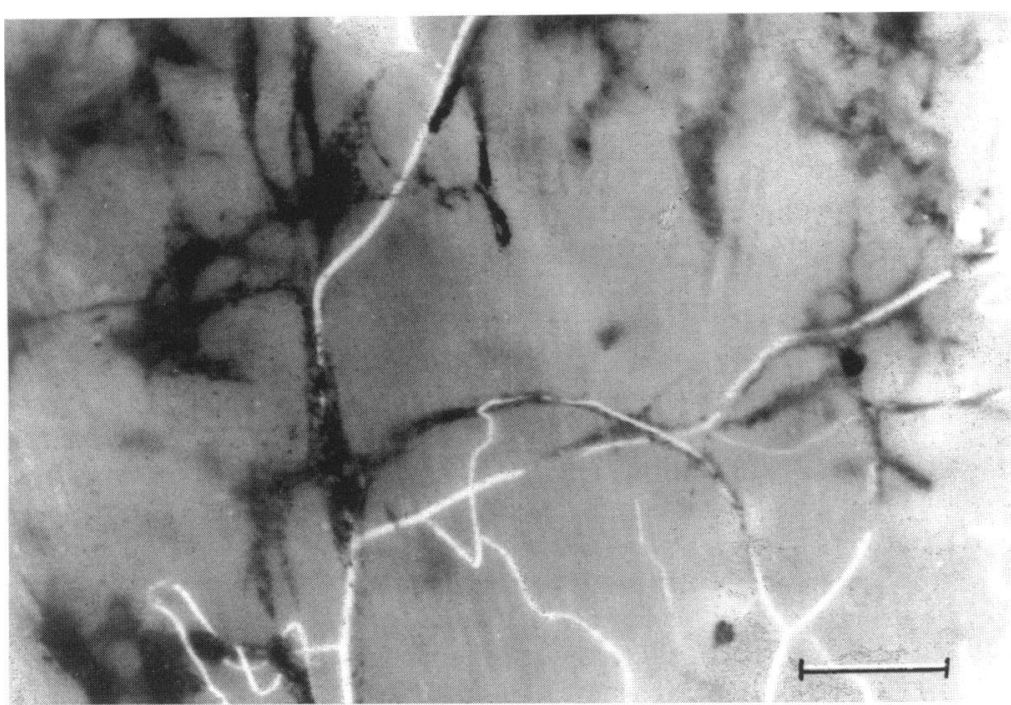

Figure 3 Retinal vessels ensheathed with hyperpigmentation in the border zone between the retinal centre and mid periphery. Bar $=250 \mu \mathrm{m}$.

hyperpigmentation, and occasionally PAS positive material occluded a lumen narrowed by hyperpigmented material.

The only conspicuous hyperpigmentation in the deeper retinal layers was patchy hyperplasia of the retinal pigment epithelium occurring in the retinal mid periphery and periphery probably accounting for the deeper lying hyperpigmented spicules seen in the ocular background.

\section{Discussion}

It is a matter of dispute which signs in the clinical spectrum of the Bardet-Biedl syndrome are necessary and sufficient to establish the diagnosis in borderline cases, since some phenotypical variation exists. In the present case the phenotype was typical of BBS, despite the absence of polydactyly. Additionally, the diagnosis was indirectly supported by broad hands with short fingers, early involvement of the central retina, and renal failure.

Current evidence suggests that the primary event in the pathogenesis of BBS is degenerative loss of retinal photoreceptors, with secondary involvement of the inner retinal

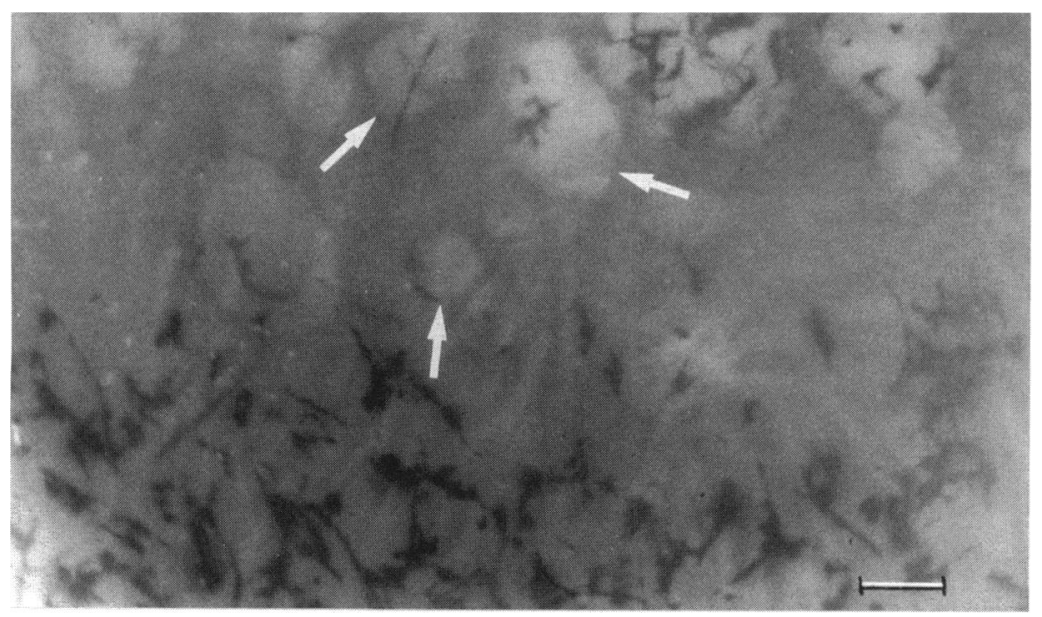

Figure 4 The transition zone from the retinal mid periphery with many bony spicules (bottom) to the greyish looking periphery with sparse spicules (top). Deep stamped out lesions are seen (arrows). Bar $=750 \mu \mathrm{m}$. layers and the retinal pigment epithelium. This evidence is supported by histopathological studies, ${ }^{6} 711$ and by electrophysiological findings of reduced or absent ERG responses from retinal photoreceptors, and frequently occurring EOG changes indicating damage to the pigment epithelium. ${ }^{12-14}$ The findings of the present study are in accordance with this evidence. As judged from the clinical history the examined eye had reached a rather advanced stage of retinal dystrophy, and this could be confirmed by the histological findings of pronounced degeneration in all areas of the retina and the choroid. The clinical history with normal blood pressure makes it unlikely that the vascular pathology should be due to arterial hypertension.

A striking feature of the examined eye was the lack of cast vessels in the retinal mid periphery displaying bone spicules characteristic of retinal dystrophy. The bone spicules probably represent melanin from retinal pigment epithelial cells migrated into the inner retina. This pattern of pigment migration is a response common to various vascular, inflammatory, and dystrophic disease processes. However, in retinitis pigmentosa the migrated pigment displays a characteristic ring shaped distribution in the retinal mid periphery. It is generally assumed that this geographical predilection reflects the fact that photoreceptor degeneration begins in this area. However, it might be anticipated that photoreceptor loss outside the mid peripheral retinal zone would have a similar potential for inducing pigment epithelial migration as it would have in the mid peripheral zone. The different response patterns in different retinal areas might perhaps be due to some regional variation in retinal anatomy or physiology, the nature of which is presently unknown.

The generalised thinning of retinal vessels seen ophthalmoscopically in retinitis pigmentosa could also be recognised in the cast retinal vessels of the eye examined here. An exact measure of vascular thickness would be invalid, however, because of variable artefactual shrinkage of the casting material. In the examined case the transition from the central area with cast vessels to the mid peripheral area with bone spicules was rather abrupt, with only few border zone vessels both containing casting material and being ensheathed with pigment granules. One might, therefore, suggest that vascular occlusion was in some way related to the accumulation of pigment material in and around retinal vessels. The exact nature of such a relation remains to be elucidated, however.

The loss of three dimensional structure of vessels in the macular retina was seen histologically to be due to occlusion of the external retinal capillary layers. This is in accordance with the presumption that photoreceptor degeneration will mainly lead to decreased metabolic demand in outer retinal layers. It is notable, however, that any vessels remained in the macular retina at all. As judged from the histological appearance, the degenerative process of the retinal tissue had almost reached 


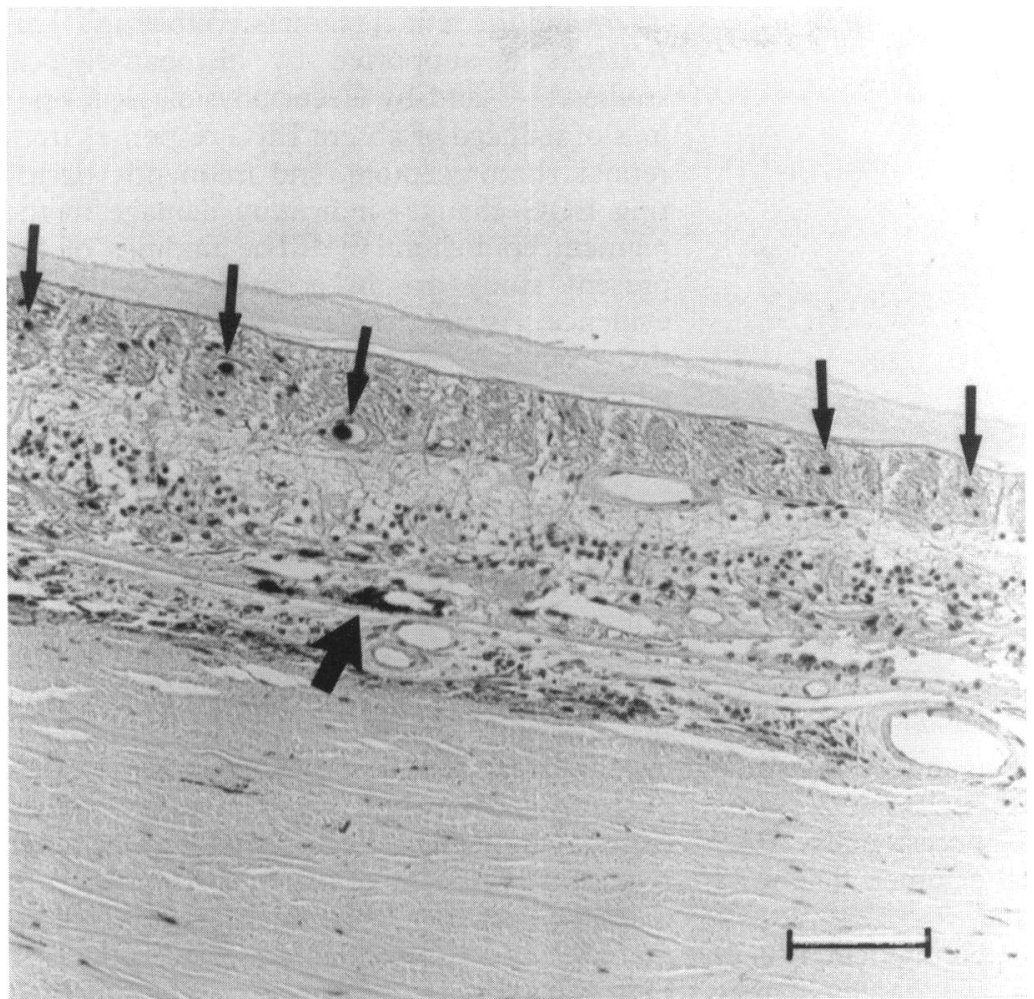

Figure 5 Haematoxylin and eosin stained section of the macular retina. Shrunken casting material is seen inside several retinal vessels all localised in the same retinal layer (small arrows). An area with pigment epithelial hyperplasia is seen at the junction towards the choroid (large arrow). Bar $=100 \mu \mathrm{m}$.

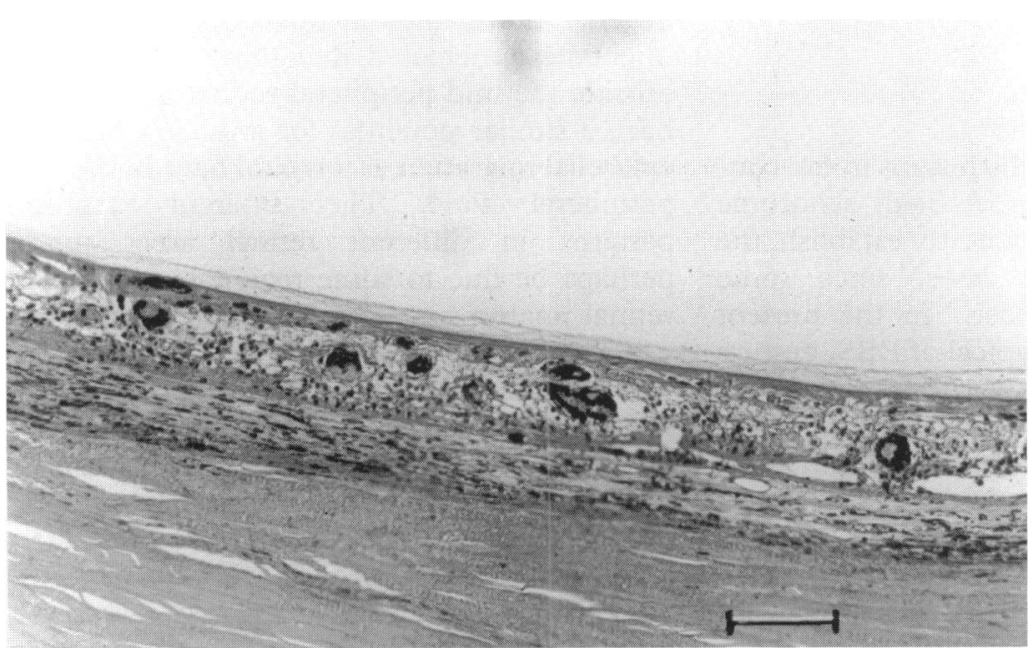

Figure 6 Periodic acid Schiff and haematoxylin stained section of the retinal mid periphery. Retinal vessels are seen to be ensheathed with hyperpigmented granular material protruding into the vascular lumen in a varying degree. The remaining parts of the lumen are occluded by periodic acid Schiff positive material. Bar $=100 \mu \mathrm{m}$.

the same degree of severity in all retinal areas. Therefore, the persistence of retinal vessels in the macular area seems peculiar. This finding could perhaps be due to the fact that the pathophysiological process involved in the remodelling of retinal vascular structure had not reached its final level, or that the remodelling process had been unable to progress to a degree where macular blood flow was totally eliminated.

It is often assumed that the superior and inferior temporal retinal arcade vessels are end vessels, with no anastomoses connecting the two. The identification of such anastomoses is difficult in normals because of the dense distribution of retinal vessels along the temporal raphe, especially in the macular area. However, with the casting technique employed in the present study, frequent anastomoses at the capillary level have been found between the upper and the lower temporal arcade along the temporal raphe in normals (unpublished). Thus, the anastomoses found between the upper and the lower temporal arcade in the present study may be the result of an adaptive remodelling of anastomoses normally present, which have become recognisable because of the pronounced decrease in vascular density in the macular area.

The artefact of casting material extravasating diffusely into the retinal tissue around the optic nerve head is seen in normal eyes when the casting material is introduced into the central retinal artery under highest possible pressure. The presence of this artefact may therefore be due to increased flow resistance in the diminished retinal vascular bed of the cast eye. The extravasation of the casting material around the fovea, however, is peculiar and has not been experienced before with the employed casting technique. It is possible that the perifoveal capillaries may have been especially vulnerable to the casting pressure transmitted to the capillary level. Such a vulnerability might perhaps be related to the cystoid degeneration noticed in this area histologically, and might be part of a macular dystrophy.

In conclusion, the present case report has shown an abnormal distribution and structure of retinal vessels in an advanced case of the Bardet-Biedl syndrome. The findings may contribute to better understanding of adaptive properties of the retinal vasculature in retinal dystrophies.

The skilful assistance of technicians Birthe Olesen and Ena Kristensen is gratefully acknowledged.

1 Schachat AP, Maumenee IH. Bardet-Biedl syndrome and related disorders. Arch Ophthalmol 1982; 100 285-8.

2 Riise R. Visual function in Laurence-Moon-Bardet-Biedl syndrome. A survey of 26 cases. Acta Ophthalmol 1987; 65 (suppl): 128-31.

3 Haim M. Prevalence of retinitis pigmentosa and allied disorders in Denmark. II. Systemic involvement and age at onset. Acta Ophthalmol 1992; 70: 417-26.

4 Campo RV, Aaberg TM. Ocular and systemic manifestations of the Bardet-Biedl syndrome. Am $\mathcal{F}$ Ophthalmol 1982; 94: 750-6.

5 Leys MJ, Schreiner LA, Hansen RM, Mayer DL, Fulton $\mathrm{AB}$. Visual acuities and dark-adapted thresholds of children with Bardet-Biedl syndrome. Am f Ophthalmol 1988; 106: 561-9.

6 Brattgård S-O. The pathology of Laurence-Moon-Biedl syndrome. Acta Pathol Microbiol Scand 1949; 26: 525-37.

7 McLoughlin TG, Shanklin DR. Pathology of the LaurenceMoon-Bardet-Biedl syndrome. $\mathcal{F}$ Pathol Bacteriol 1967; 93: 65-79.

8 Stanescu B, Nereantu F. Laurence-Moon-Bardet-Bied syndrome with juvenile macular degenerescence Stargardt. Ophthalmologica 1971;162: 76-81.

9 Runge P, Calver D, Marshall J, Taylor D. Histopathology of mitochondrial cytopathy and the Laurenceof mitochondrial cytopathy and the LaurenceMoon-B

10 Bek T, Jensen PK. Three-dimensional structure of human retinal vessels studied by vascular casting. Acta Ophthalmol 1993; 71: 506-13.

11 Bisland T. The Laurence-Moon-Bardet-Biedl syndrome. Report of a typical case with complete necropsy. $A m \tilde{J}$ Ophthalmol 1951; 34: 874-84.

12 Katsumi O, Tanino T, Hirose T, Larson EW, Skladzien CJ. Laurence-Moon-Bardet-Biedl syndrome: electrophysiological and psychophysical findings. Ipn 7 Ophthalmol 1985; 29: 282-9.

13 Harrison JM, Van Heuven WAJ. Rod-cone interaction in the ERG of a patient with Bardet-Biedl syndrome. Doc Ophthalmol 1985; 60: 203-9.

14 Rizzo JF, Berson EL, Lessell S. Retinal and neurologic findings in the Laurence-Moon-Bardet-Biedl phenotype. Ophthalmology 1986; 93: 1452-6. 